\title{
Spectral phase conjugation by quasi-phase-matched three-wave mixing
}

\author{
Mankei Tsang *, Demetri Psaltis \\ Department of Electrical Engineering, California Institute of Technology, 1200 E. California Blvd., Pasadena, CA 91125, USA
}

Received 10 June 2004; received in revised form 22 July 2004; accepted 3 September 2004

\begin{abstract}
A novel spectral phase conjugation scheme by three-wave mixing is proposed. It is shown that a phase-conjugated and time-reversed replica of the incoming signal can be generated, if appropriate quasi-phase matching is achieved and the three-wave mixing process is transversely pumped by a short second-harmonic pulse.
\end{abstract}

(c) 2004 Elsevier B.V. All rights reserved.

Keywords: Phase conjugation; Time reversal; Quasi-phase matching; Three-wave mixing

\section{Introduction}

Conventional phase conjugation schemes perform the so-called phase conjugation with spectral inversion [1]. The output of such schemes has a spectrum that is phase conjugated and spectrally inverted with respect to the input. In time domain, this is equivalent to phase conjugation of the pulse envelope, so the scheme is also called temporal phase conjugation (TPC) [2]. A distinct kind of phase conjugation, which performs phase

\footnotetext{
${ }^{*}$ Corresponding author. Tel.: +1 626395 3888; fax: +1 626 5688437.

E-mail address: mankei@sunoptics.caltech.edu (M. Tsang).
}

conjugation without spectral inversion, is first suggested by Miller [3]. The output spectrum of such a phase conjugation scheme is the phase conjugation of the input spectrum, and the scheme is therefore called spectral phase conjugation (SPC). In time domain, the output pulse envelope is the phase conjugation and time reversal of the input pulse envelope.

While TPC can compensate for even-order dispersion [1], self-phase modulation [4] and intrapulse Raman scattering [5], SPC can simultaneously compensate for all chromatic dispersion [6], self-phase modulation and self-steepening [2], thus making it attractive for ultrafast applications such as optical communications. The time reversal 
operation associated with SPC is also useful for signal processing [7].

The physical implementation of SPC is first suggested by Miller using short-pump four-wave mixing (FWM) [3], and later demonstrated, for example, using photon echo [8,9], spectral hole burning $[10,11]$, temporal holography [6], spectral holography [12] and spectral three-wave mixing (TWM) [13]. The FWM scheme is especially appealing to real-world applications due to its simple setup, and its efficiency is recently shown to be significantly higher than previously considered [14]. However, the FWM scheme requires accurate synchronization of two short pump pulses, which may undermine its robustness. A setup that combines the elegant geometry of Miller's scheme and the robustness of TWM can therefore be advantageous. It is also of fundamental interest to investigate if the use of second-order nonlinearity can provide a higher efficiency than the FWM configuration.

\section{Configuration}

The proposed configuration is shown in Fig. 1. It is similar to surface-emitting second-harmonic generation $[15,16]$, transverse-pumping parametric amplification [17] and transverse-pumping phase conjugation [18]. The difference in our proposed system is that the pump pulse $A_{\mathrm{p}}(t)$ is much shorter than the signal pulse $A_{\mathrm{s}}(t)$. Therefore, the pump

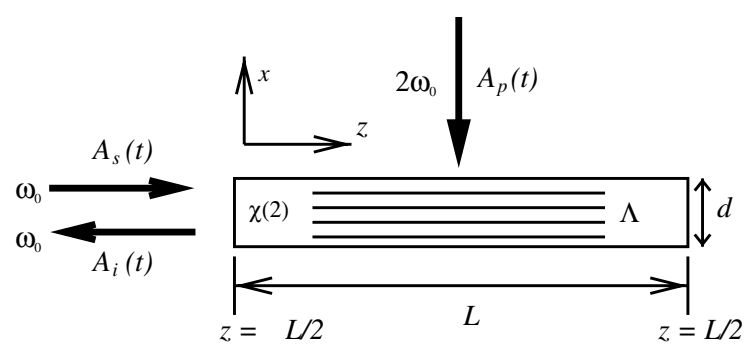

Fig. 1. Geometry of SPC by quasi-phase-matched three-wave mixing. $A_{\mathrm{s}}(t)$ is the incoming signal pulse with a carrier frequency $\omega_{0}$, and $A_{\mathrm{p}}(t)$ is the second-harmonic pump pulse. $A_{\mathrm{i}}(t)$ is the generated idler pulse. Quasi-phase matching is achieved by a $\chi^{(2)}$ grating with period $\Lambda$ along $x$. pulse takes a "snapshot" of the signal pulse, and when phase matching is satisfied, the generated idler pulse $A_{\mathrm{i}}(t)$ is a backward-propagating, phaseconjugated and time-reversed replica of the input signal pulse. The second-harmonic pump pulse can be generated by conventional secondharmonic generation methods.

\section{Theory}

To achieve phase matching we must have

$\mathbf{k}_{\mathrm{s}}+\mathbf{k}_{\mathrm{i}}=\mathbf{k}_{\mathrm{p}}+\mathbf{K}$,

where $\mathbf{k}_{\mathrm{i}}$ is the wave vector of the idler, $\mathbf{k}_{\mathrm{s}}$ is the wave vector of the signal, $\mathbf{k}_{\mathrm{p}}$ is the wave vector of the pump, and $\mathbf{K}=2 \pi / \Lambda \hat{\mathbf{x}}$ is the $\chi^{(2)}$ grating vector. In our geometry $\mathbf{k}_{\mathrm{s}}=-\mathbf{k}_{\mathrm{i}}$, so $\mathbf{K}=-\mathbf{k}_{\mathrm{p}}$ or

$\Lambda=\frac{\lambda_{0}\left(2 \omega_{0}\right)}{n\left(2 \omega_{0}\right)}$,

where $\lambda_{0}\left(2 \omega_{0}\right)$ is the free-space wavelength at $2 \omega_{0}$ and $n\left(2 \omega_{0}\right)$ is the refractive index at $2 \omega_{0}$. In other words, the grating period should be equal to the second-harmonic wavelength in the medium, which is in general sub-micron. A $\chi^{(2)}$ grating with such a small period can be fabricated, for example, in $\mathrm{AlGaAs} / \mathrm{GaAs}$ heterostructures [16,19], asymmetric coupled quantum wells [20], poled-polymer waveguides [21] and KTP crystals [22].

Notice that in our scheme the $\chi^{(2)}$ grating is only used to cancel the carrier wave vector of the pump pulse, while the additional wave vector due to the broad bandwidth of the pump pulse is accounted for in the coupled-mode formalism below. Provided that such quasi-phase matching is achieved, the coupled-mode equations of pulse envelopes $A_{\mathrm{p}}$, $A_{\mathrm{s}}$ and $A_{\mathrm{i}}$ can be derived from the wave equation, and are given by:

$$
\begin{aligned}
& -\frac{\partial A_{\mathrm{p}}}{\partial x}+\frac{1}{v_{x}} \frac{\partial A_{\mathrm{p}}}{\partial t}=2 \mathrm{j} \gamma \frac{n_{0}}{n\left(2 \omega_{0}\right)} A_{\mathrm{s}} A_{\mathrm{i}}, \\
& \frac{\partial A_{\mathrm{q}}}{\partial x}+\frac{1}{v_{x}} \frac{\partial A_{\mathrm{q}}}{\partial t}=2 \mathrm{j} \gamma \frac{n_{0}}{n\left(2 \omega_{0}\right)} A_{\mathrm{s}} A_{\mathrm{i}}, \\
& \frac{\partial A_{\mathrm{s}}}{\partial z}+\frac{1}{v} \frac{\partial A_{\mathrm{s}}}{\partial t}=\mathrm{j} \gamma\left(A_{\mathrm{p}}+A_{\mathrm{q}}\right) A_{\mathrm{i}}^{*},
\end{aligned}
$$


$-\frac{\partial A_{\mathrm{i}}}{\partial z}+\frac{1}{v} \frac{\partial A_{\mathrm{i}}}{\partial t}=\mathrm{j} \gamma\left(A_{\mathrm{p}}+A_{\mathrm{q}}\right) A_{\mathrm{s}}^{*}$

$\gamma=\frac{\omega_{0} \chi^{(2)}}{2 c n_{0}}$

where $A_{\mathrm{q}}$ is the upward-propagating secondharmonic wave, which can be neglected in general but included here for completeness, $v_{x}$ is the group velocity at $2 \omega_{0}$ along $x, v$ is the group velocity at $\omega_{0}$ along $z$, and $n_{0}$ is the refractive index at $\omega_{0}$. Diffraction and group-velocity dispersion are neglected.

If a waveguide structure is used, then $\gamma$ should also include an overlapping factor that describes the overlapping extent of the signal and idler waveguide modes

$\gamma=\frac{\omega_{0} \chi^{(2)}}{2 c n_{0}} \int \mathrm{d} \mathbf{r}_{\perp} \psi_{\mathrm{s}} \psi_{\mathrm{i}}$

where $\psi_{\mathrm{s}}$ and $\psi_{\mathrm{i}}$ are the normalized waveguide mode profiles of the signal and the idler, respectively.

If the right-hand sides of Eqs. (3) and (4) are much smaller than the left-hand sides, then the pump can be assumed to be undepleted. Moreover, we can neglect the $x$ dimension if the pump pulse spatial width $v_{x} T_{\mathrm{p}}$ is much longer than the thickness of the medium $d$. The resulting coupled-mode equations of $A_{\mathrm{s}}$ and $A_{\mathrm{i}}$ are:

$v \frac{\partial A_{\mathrm{s}}(z, t)}{\partial z}+\frac{\partial A_{\mathrm{s}}(z, t)}{\partial t}=\mathrm{j} g(t) A_{\mathrm{i}}^{*}(z, t)$,

$-v \frac{\partial A_{\mathrm{i}}(z, t)}{\partial z}+\frac{\partial A_{\mathrm{i}}(z, t)}{\partial t}=\mathrm{j} g(t) A_{\mathrm{s}}^{*}(z, t)$,

$g(t)=\gamma v A_{\mathrm{p}}(t)$.

Eqs. (9) and (10) are completely identical to the equations that describe the signal and the idler in the FWM geometry [14]. The boundary conditions are assumed to be:

$A_{\mathrm{s}}\left(-\frac{L}{2}, t\right)=F\left(t+\frac{L}{2 v}\right)$,

$A_{\mathrm{i}}\left(\frac{L}{2}, t\right)=0$.
Assuming that $g(t)=|g(t)| \exp (\mathrm{j} \theta), \theta$ is constant, and the pulse width of $A_{\mathrm{p}}$ is much shorter than that of $A_{\mathrm{s}}$, Eqs. (9) and (10) can be solved using the same method described in [14]. The solution is then given by:

$$
\begin{aligned}
A_{\mathrm{s}}(z, t)= & F\left(t-\frac{z}{v}\right) \cosh \left[\int_{-\infty}^{t}\left|g\left(t^{\prime}\right)\right| \mathrm{d} t^{\prime}\right], \\
A_{\mathrm{i}}(z, t)= & \mathrm{j} F^{*}\left(-t-\frac{z}{v}\right) \exp (\mathrm{j} \theta) \\
& \times \sinh \left[\int_{-\infty}^{t}\left|g\left(t^{\prime}\right)\right| \mathrm{d} t^{\prime}\right] .
\end{aligned}
$$

The idler exiting at $z=-L / 2$ is

$$
\begin{aligned}
A_{\mathrm{i}}\left(-\frac{L}{2}, t\right)= & \mathrm{j} F^{*}\left(-t+\frac{L}{2 v}\right) \exp (\mathrm{j} \theta) \\
& \times \sinh \left[\int_{-\infty}^{\infty}\left|\gamma v A_{\mathrm{p}}\left(t^{\prime}\right)\right| \mathrm{d} t^{\prime}\right] .
\end{aligned}
$$

The conversion efficiency, defined as the input signal energy divided by the output idler energy, is

$$
\begin{aligned}
\eta & \equiv \frac{\int_{-\infty}^{\infty}\left|A_{\mathrm{i}}\left(-\frac{L}{2}, t^{\prime}\right)\right|^{2} \mathrm{~d} t^{\prime}}{\int_{-\infty}^{\infty}\left|A_{\mathrm{s}}\left(-\frac{L}{2}, t^{\prime}\right)\right|^{2} \mathrm{~d} t^{\prime}} \\
& =\sinh ^{2}\left[\int_{-\infty}^{\infty}\left|\gamma v A_{\mathrm{p}}\left(t^{\prime}\right)\right| \mathrm{d} t^{\prime}\right] .
\end{aligned}
$$

Similar to the FWM configuration, the following conditions should be satisfied for accurate SPC operation:

$\frac{L}{v} \gg T_{\mathrm{s}} \gg T_{\mathrm{p}} \gg \frac{d}{v_{x}}$,

where $T_{\mathrm{s}}$ is the pulse width of the signal.

\section{Comparison with the FWM scheme}

To compare the efficiency of the TWM scheme with that of the FWM scheme, we first compute the pump energy $E_{\mathrm{p}}$ required to achieve a certain conversion efficiency $\eta$ by assuming that the pump pulse is a Gaussian

$A_{\mathrm{p}}(t)=A_{\mathrm{p} 0} \exp \left(-\frac{t^{2}}{2 T_{\mathrm{p}}^{2}}\right)$, 
$v=c / n_{0}$, and the cross-section area of the pump beam is $L d . E_{\mathrm{p}}$ is then given by:

$$
\begin{aligned}
E_{\mathrm{p}} & =\frac{n_{0} L d}{2 \eta_{0}} \int\left|A_{\mathrm{p}}\left(t^{\prime}\right)\right|^{2} \mathrm{~d} t^{\prime}, \\
& =\frac{n_{0}^{5} L d}{\sqrt{\pi} \eta_{0} \omega_{0}^{2}\left[\chi^{(2)}\right]^{2} T_{\mathrm{p}}}\left[\sinh ^{-1}(\sqrt{\eta})\right]^{2},
\end{aligned}
$$

where $\eta_{0}$ is the free-space impedance. Compare this with the total pump energy required for the FWM configuration, assuming that the two pump pulses are identical:

$$
\begin{aligned}
E_{\mathrm{p}}^{\prime} & =\frac{4 n_{0}^{\prime 3} L d}{3 \eta_{0} \omega_{0} \chi^{(3)}} \sinh ^{-1}(\sqrt{\eta}), \\
& =\frac{n_{0}^{\prime} L d}{\omega_{0} n_{2}} \sinh ^{-1}(\sqrt{\eta}),
\end{aligned}
$$

where $n_{0}^{\prime}$ is the refractive index of the $\chi^{(3)}$ medium. The TWM scheme is thus more efficient when

$$
\left[\frac{\sqrt{\pi}}{\sinh ^{-1}(\sqrt{\eta})}\right]\left(\omega_{0} T_{\mathrm{p}}\right) \frac{\eta_{0}\left[\chi^{(2)}\right]^{2}}{n_{0}^{5}}>\frac{n_{2}}{n_{0}^{\prime}} .
$$

For example, for $\lambda_{0}=800 \mathrm{~nm}, T_{\mathrm{p}}=100 \mathrm{fs}$, a $\mathrm{GaAs} / \mathrm{AlGaAs}$ heterostructure with $\chi^{(2)} \approx 50 \mathrm{pm} /$ $\mathrm{V}, n_{0} \approx 3, \eta=100 \%$, the left-hand side of Eq. (24) is about $10^{-14} \mathrm{~cm}^{2} / \mathrm{W}$, which is close to the $n_{2}$ of $\mathrm{CS}_{2}$, but much lower than that of conjugated polymers $\left(\sim 10^{-11} \mathrm{~cm}^{2} / \mathrm{W}[23]\right)$. That said, $\chi^{(2)}$ of asymmetric coupled GaAs/AlAs quantum wells can theoretically reach $30 \mathrm{~nm} / \mathrm{V}$ in the far infrared regime [24], potentially giving rise to a much lower pump energy requirement. The TWM scheme also eliminates the need of the second pump pulse and avoids the difficulty in synchronizing two ultrashort pulses in a thin medium.

\section{Numerical analysis}

In order to confirm the validity of the approximations in our theoretical predictions, we perform numerical simulations of Eqs. (9) and (10), using GaAs/AlGaAs heterostructure as the nonlinear medium, a Gaussian pump pulse with $T_{\mathrm{p}}=100 \mathrm{fs}$ and pump energy $E_{\mathrm{p}}=2.1 \mu \mathrm{J}$. The incoming signal is assumed to be

$$
\begin{aligned}
F(t)= & A_{\mathrm{s} 0}\left\{-\exp \left[-\frac{1+\mathrm{j}}{2}\left(\frac{t+2 T_{\mathrm{s}}}{T_{\mathrm{s}}}\right)^{2}\right]\right. \\
& \left.+\frac{1}{2} \exp \left[-\frac{1}{2}\left(\frac{t-2 T_{\mathrm{s}}}{T_{\mathrm{s}}}\right)^{2}\right]\right\}
\end{aligned}
$$

with $T_{\mathrm{s}}=1 \mathrm{ps}$. The calculated conversion efficiency is $100 \%$, consistent with the theoretical efficiency from Eq. (17). The signal and idler pulse shapes from the numerical analysis are plotted in Fig. 2, which confirms that SPC can indeed be accurately performed by TWM. The required pump energy $2.1 \mu \mathrm{J}$ is much higher than that theorized in [14] for the FWM scheme using polydiacetylene $(\sim 10 \mathrm{~nJ})$, but it is still much lower than the pump energy used in [13] $(\sim 1 \mathrm{~mJ})$.

Using the same parameters, Fig. 3 plots the theoretical conversion efficiency calculated from Eq. (17) and that obtained from numerical analysis against the pump energy. The numerical results agree quite well with the theoretical prediction, although the former is slightly lower, due to slight deviation from the ideal conditions stated in Eq. (18).

\section{Competing third-order nonlinearity}

With a high pump intensity, competing thirdorder nonlinearity in the form of cross-phase modulation (XPM) can be detrimental to the SPC accuracy and efficiency in the same manner as for the FWM scheme [14]. One way to control $\mathrm{XPM}$ is by the same XPM compensation method described in [14], which uses the wave mixing process to introduce phase variations to the signal and the idler, so that the Kerr phase modulation due to the strong pump can be exactly cancelled. The theoretical proof is very similar to the FWM case [14], and the pump phase adjustment is

$\theta(t)=\theta_{0}+\int_{-\infty}^{t} \frac{3 \omega_{0} v \chi^{(3)}}{2 c n_{0}}\left|A_{\mathrm{p}}\left(t^{\prime}\right)\right|^{2} \mathrm{~d} t^{\prime}$.

This phase adjustment is an almost linear function of time, or equivalently a center frequency shift. Therefore, if this compensation method is used, the $\chi^{(2)}$ grating period should also be adjusted to account for the wave vector change due to the center frequency shift. 

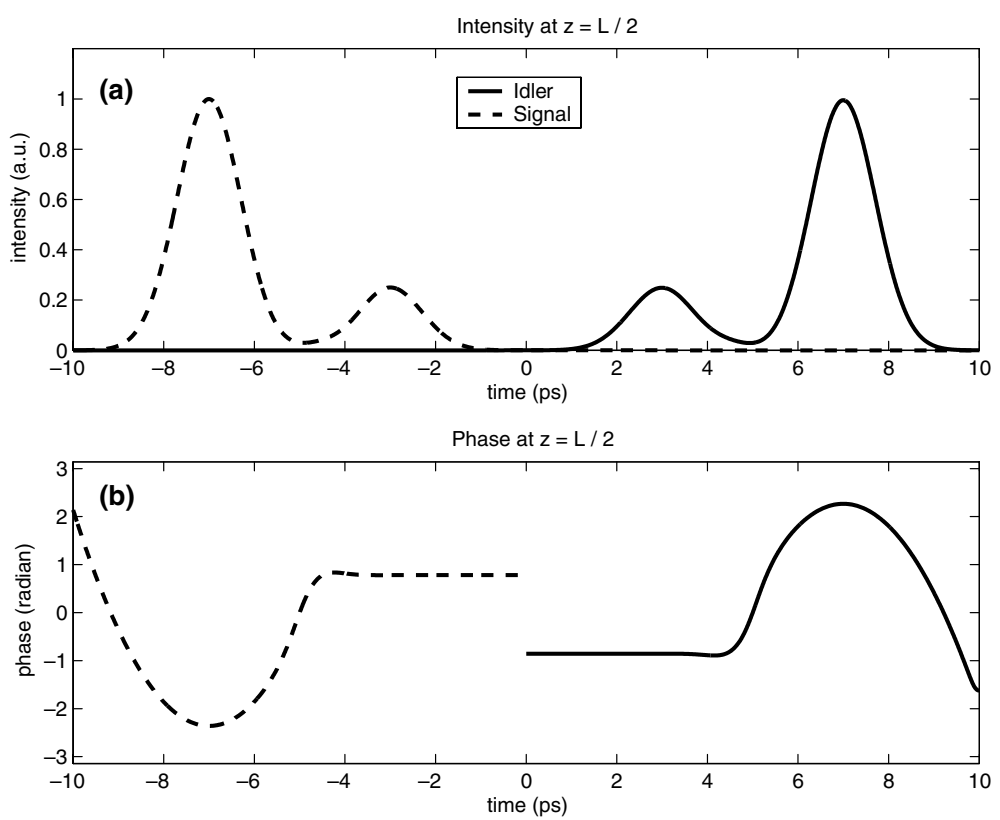

Fig. 2. Plots of intensity and phase of incoming signal and output idler from numerical analysis. It is clear from the plots that the idler is a phase-conjugated and time-reversed replica of the signal, confirming our theoretical derivations. Parameters used are $\chi^{(2)}=50 \mathrm{pm} /$ $\mathrm{V}, n_{0}=3, L=1 \mathrm{~mm}, d=5 \mu \mathrm{m}$, width in $y=d, E_{\mathrm{p}}=2.1 \mu \mathrm{J}$, pump fluence $=E_{\mathrm{p}} / L d$. For such dimensions waveguide confinement of the signal and the idler is necessary.

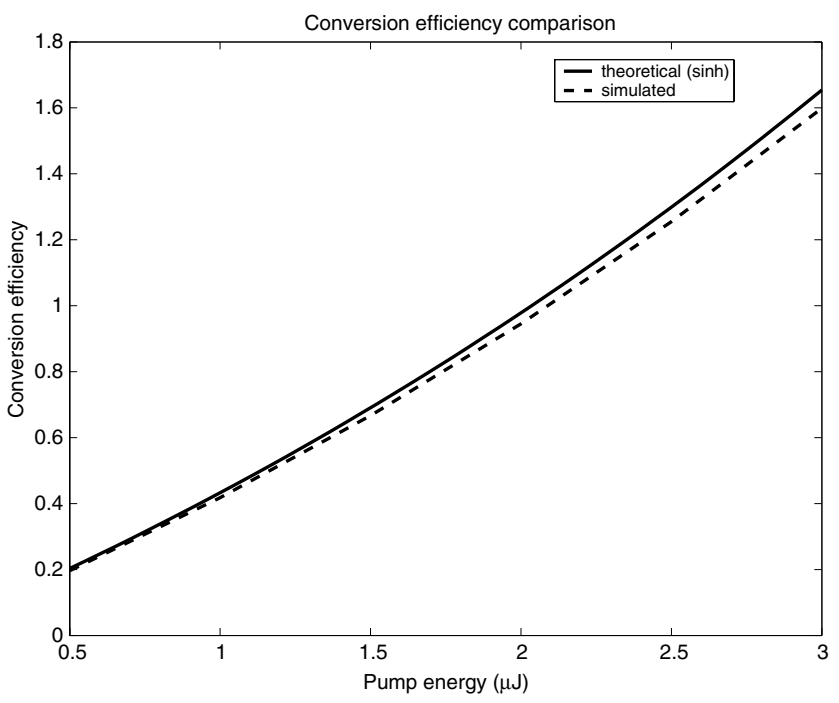

Fig. 3. Theoretical conversion efficiency derived from Eq. (17) and that from numerical analysis plotted against pump energy. See caption of Fig. 2 for parameters used. 


\section{Conclusion}

We have proposed a novel SPC scheme by the use of quasi-phase-matched TWM. The advantages of the TWM scheme over the FWM scheme include the elimination of the second pump pulse, higher conversion efficiency for certain parameters and possible independent management of secondorder and third-order nonlinearities. Numerical results confirm our theoretical predictions, and experimental verification will be the focus of our future work.

\section{Acknowledgements}

This work was supported in part by the Engineering Research Centers Program of the National Science Foundation under Award Number EEC9402726 and by the DARPA Center for OptoFluidic Integration at Caltech.

\section{References}

[1] A. Yariv, D. Fekete, D.M. Pepper, Opt. Lett. 4 (1979) 52.

[2] M. Tsang, D. Psaltis, Opt. Lett. 28 (2003) 1558.

[3] D.A.B. Miller, Opt. Lett. 5 (1980) 300.

[4] R.A. Fisher, B.R. Suydam, D. Yevick, Opt. Lett. 8 (1983) 611.

[5] S. Chi, S.F. Wen, Opt. Lett. 19 (1994) 1705.

[6] C. Joubert, M.L. Roblin, R. Grousson, Appl. Opt. 28 (1989) 4604.
[7] D.M. Marom, D. Panasenko, P. Sun, Y.T. Mazurenko, Y. Fainman, IEEE J. Sel. Top. Quantum Electron. 7 (2001) 683.

[8] N.W. Carlson, L.J. Rothberg, A.G. Yodh, W.R. Babbitt, T.W. Mossberg, Opt. Lett. 8 (1983) 483.

[9] V.L. da Silva, Y. Silberberg, J.P. Heritage, E.W. Chase, M.A. Saifi, M.J. Andrejco, Opt. Lett. 16 (1991) 1340.

[10] A. Rebane, J. Aaviksoo, J. Kuhl, Appl. Phys. Lett. 54 (1989) 93.

[11] S. Fraigne, J.P. Galaup, J.L. Le Gouet, B. Bousquet, L. Canioni, M. Joffre, J.P. Likforman, J. Opt. Soc. Am. B 20 (2003) 1555.

[12] A.M. Weiner, D.E. Leaird, D.H. Reitze, IEEE J. Quantum Electron. 28 (1992) 2251.

[13] D. Marom, D. Panasenko, R. Rokitski, P. Sun, Y. Fainman, Opt. Lett. 25 (2000) 132.

[14] M. Tsang, D. Psaltis, Opt. Express 12 (2004) 2207.

[15] R. Normandin, G.I. Stegeman, Opt. Lett. 4 (1979) 58.

[16] D. Vakhshoori, R.J. Fischer, M. Hong, D.L. Sivco, G.J. Zydzik, G.N.S. Chu, A.Y. Cho, Appl. Phys. Lett. 59 (1991) 896.

[17] Y.J. Ding, S.J. Lee, J.B. Khurgin, Phys. Rev. Lett. 75 (1995) 429.

[18] Y.J. Ding, J.B. Khurgin, S.J. Lee, Opt. Quantum Electron. 28 (1996) 1617.

[19] C. Degen, G. Jennemann, I. Fischer, W. Elsaber, S. Leu, R. Rettig, W. Stolz, Opt. Quantum Electron. 34 (2002) 707.

[20] S. Janz, F. Chatenoud, R. Normandin, Opt. Lett. 19 (1994) 622.

[21] A. Otomo, G.I. Stegeman, M.C. Flipse, M.B.J. Diemeer, W.H.G. Horsthuis, G.R. Mohlmann, J. Opt. Soc. Am. B 15 (1998) 759.

[22] C. Canalias, V. Pasiskevicius, R. Clemens, F. Laurell, Appl. Phys. Lett. 82 (2003) 4233.

[23] R. Quintero-Torres, M. Thakur, J. Appl. Phys. 85 (1999) 401.

[24] S. Li, J. Khurgin, Appl. Phys. Lett. 62 (1993) 1727. 